\title{
Urban plant phenology monitoring: Expanding the functions of widespread surveillance cameras to nature rhythm understanding
}

\author{
Lijun Deng ${ }^{\mathrm{a}, \mathrm{b}}$, Yi Lin ${ }^{\mathrm{a}, *}$, Lei Yan ${ }^{\mathrm{a}}$, Solomon Tesfamichael ${ }^{\mathrm{c}}$, Roland Billen ${ }^{\mathrm{d}}$, Yunjun Yao ${ }^{\mathrm{e}}$, Wei Yao ${ }^{\mathrm{f}}$, \\ Xiuwan Chen ${ }^{\mathrm{a}}$, Xing Fang ${ }^{\mathrm{g}}$, Cheng Wang ${ }^{\mathrm{h}}$, Xin Jing ${ }^{\mathrm{a}}$ \\ ${ }^{a}$ Institute of Remote Sensing and GIS, Beijing Key Lab of Spatial Information Integration and Its Applications, School of Earth and Space Sciences, Peking University, \\ Beijing, 100871, China \\ ${ }^{\mathrm{b}}$ School of Earth Sciences and Resources, China University of Geosciences, Beijing, 100083, China \\ ${ }^{\mathrm{c}}$ Department of Geography, Environment Management \& Energy Studies, University of Johannesburg, Johannesburg, South Africa \\ ${ }^{\mathrm{d}}$ Department of Geography, University of Liège, Liège, 4000, Belgium \\ ${ }^{\text {e }}$ School of Geography, Beijing Normal University, Beijing, 100875, China \\ ${ }^{\mathrm{f}}$ Department of Land Surveying and Geo-Informatics, The Hong Kong Polytechnic University, Kowloon, Hong Kong \\ ${ }^{g}$ School of Geodesy and Geomatics, Wuhan University, Wuhan, Hubei, China \\ ${ }^{\mathrm{h}}$ Institute of Remote Sensing and Digital Earth, Chinese Academy of Sciences, Beijing, 100094, China
}

\section{A R T I C L E I N F O}

\section{Keywords:}

Urban plant phenology monitoring

Surveillance camera

Software plug-in

Phenology model

Phenological index

\begin{abstract}
A B S T R A C T
Phenology is an important ecological indicator for understanding the feedback of plants to climate changes, but observation of plant phenology is not a trivial task, particularly for the large-scale areas of interest. Urban plant phenology monitoring is such a typical case, since massive residents do not necessarily mean enough eligible phenology observers. To handle this traditional challenge, this study attempted those surveillance cameras (SCs) widespread almost in any city all over the world. The schematic plan is to install an automatic software module, which has the function of plant phenology monitoring, as a plug-in into any central unit that wire-controls RGB SCs. The kernel of the module is a general-purposed algorithm capable of deriving the starting and ending dates of the key phenological events of different plants that stand in the field of view of each telecontrolled SC. The kernels of the algorithm comprise deriving phenological indices from the digital number (DN) records by a SC from all of its RGB channels and, then, modeling of plant dynamics based on the proposed novel phase-limited multi-Gaussian model for curve-fitting of phenological phases. In the case of determining the key phenological dates regarding flowering and foliation in this study, tests suggested that the proposed scheme and phenological indices and the programmed software plug-in all worked well. Overall, the feasibility of using the widespread SCs for urban plant phenology monitoring was validated, and the scheme can be further extended to composing phenology observation networks at local or global scales. The solution is of implications for more understanding the interannual rhythms of terrestrial ecosystems as well as the inherent mechanisms of vegetation-climate interactions.
\end{abstract}

\section{Introduction}

Phenology serves as a crucial natural ecological bridge for learning the inherent links between plant life cycles and environmental changes such as climate changes (Koerner and Basler, 2010). As a vital branch of environment, particularly for humans, urban areas are an essential category of land surfaces concerned in the field of plant phenology. In fact, urban plant phenology has long been highlighted (Neil and Wu, 2006), as its researches facilitate better understanding of the eco-effects of both urbanization and urban heat island on plant phenological behaving (Neil and Wu, 2006; Lu et al., 2006; Neil et al., 2010; Jochner et al., 2012, 2013; Comber and Brunsdon, 2015). Furthermore, the knowledge of urban plant phenology allows for predicting future plant phenology, since cities with their amplified temperatures may serve as a proxy of future scenes (Jochner and Menzel, 2015). Hence, developing efficient techniques for urban plant phenology monitoring is becoming increasingly important.

The predominant means for plant phenology monitoring, even up to now, rely on human observation of plants at multiple stages in the phenological period (Bradley and Clarke, 2011; Jochner and Menzel,

\footnotetext{
* Corresponding author.

E-mail address: yi.lin@pku.edu.cn (Y. Lin).
} 
2015). Based on the in-field-observed plant phenological recordings for decades and even centuries, people have investigated not only the phenological problems such as reconstructions of interannual changes in past spring seasons (Rutishauser et al., 2007) and shifts in spring vegetation green-up dates across temperate biomes across China (Wu and Liu, 2013) but also the phenology-relevant issues such as net ecosystem exchange (Churkina et al., 2005), global warming (Abu-Asab et al., 2001), temperature of the medieval period in spring (Aono and Saito, 2010), and climate change (Lu et al., 2006; Wolkovich et al., 2012; Dai et al., 2013). Although this traditional strategy has proved to work well on recording the exact dates of the key phenological events of plants during their growing seasons, its extensive usages are impeded by its shortages of both intensive labor and insufficient geographic cover. Moreover, the traditional plant phenological records often cannot support reconstructing the continuous processes of historical plant dynamics. In urban environments, considerable residents do not necessarily mean enough eligible plant phenology observers. Even if enough plant phenology observers can be found, merely relying on human observations to cover thousands of cites all over the world often means an unaffordable cost.

To handle the shortcomings of field-based survey methods, spaceborne remote sensing (RS) has been widely used as a sound solution for monitoring the growths and senescence of vegetation canopies at local, regional, and global scales. The most representative cases were based on the Moderate Resolution Imaging Spectroradiometer (MODIS) RS products, which were widely applied for investigation of the spatiotemporal dynamics of various land surface phenologies (LSPs) (Zhang et al., 2003, 2004; Ahl et al., 2006; Ganguly et al., 2010). However, the applications of the satellite RS-based plant phenology monitoring plans were often restricted, in subject to the lack of adequate ground-truth data for validation of the derived results (Studer et al., 2007). Furthermore, it is hard for this kind of approaches, e.g., the BFAST package (Verbesselt et al., 2010) and TIMESAT package (Ekhlund and Jönsson, 2015), to obtain accurate, abundant, and valuable transition dates of vegetation activities due to their inadequate spatial resolutions and low re-visit frequencies (Zhang et al., 2004; Ahl et al., 2006; Ganguly et al., 2010; Studer et al., 2007). This kind of issues tend to become more serious in urban scenarios, because cities are typically filled with high buildings and scattered plants that are difficult to identify using publicly available space-borne RS data.

Another kind of methods for plant phenology monitoring is to directly measure different biochemical fluxes of plants based on the eddycovariance (EC) technology (Baldocchi et al., 2005). A number of ECbased methods have been developed to characterize spring transitions from $\mathrm{CO}_{2}$ sources to sinks for different deciduous forests (Baldocchi et al., 2005), ecosystem phenology involving spring photosynthesis in a cool temperate bog (Lafleur et al., 2005) and carbon uptake phenology for temperate and boreal deciduous forests (Gonsamo et al., 2012) as well as to calculate key LSP metrics (such as the start, end, and length of a growth season, the end of green-up, the start of brown-down, the temporal length of canopy closure, the start, end, and length of the peak, and the peak of one growth season) (Gonsamo et al., 2013). This kind of solutions can reveal the inherent mechanism of plant phenological variations, but the corresponding frame-based EC systems, even for their simplified versions, are expensive. As a result, the adoption of EC-based techniques is limited in urban plant phenology monitoring. In addition to biochemical attributes, biophysical properties such as plant structures (Calders et al., 2015) and canopy penetration (Lin and West, 2016) were also tried for plant phenology monitoring, based on static terrestrial laser scanning (TLS) (Calders et al., 2015) and mobile laser scanning (MLS) (Lin and West, 2016), respectively. However, the potential plans based on laser scanning are unaffordable for monitoring of plant phenology, whose re-visits require high temporal frequency.

Following the call-for "install video cameras to FLUXNET sites" made by Baldocchi et al. (2005), there occurred an explosive growth of using the easily-available cameras for plant phenology monitoring over the years (e.g., Richardson et al., 2007; Crimmins and Crimmins, 2008; Ahrends et al., 2009; Graham et al., 2009; Ide and Oguma, 2010; Sonnentag et al., 2011; Hufkens et al., 2012; Nijland et al., 2014; Alberton et al., 2017; Vrieling et al., 2018). The cameras used in such studies ranged from digital webcam cameras (Richardson et al., 2007) to infrared sensitive consumer-grade digital cameras (Nijland et al., 2014). The phenology monitoring modes ranged from location-fixed cameras (Crimmins and Crimmins, 2008; Ide and Oguma, 2010; Vrieling et al., 2018) to running car-based cameras (Graham et al,, 2009) and from camera-EC technical combination (Ahrends et al., 2009; Sonnentag et al., 2011) to ground-satellite data fusion (Hufkens et al., 2012; Vrieling et al., 2018). The objectives of camera-based phenology monitoring involve from forest comparisons (Zhao et al., 2012) to canopy photosynthesis predictions (Toomey et al., 2015). The proposed phenological indices include 2G_RBi (the difference of the divergence of both red from green and blue from green, using absolute channel brightness) (Richardson et al., 2007), GCC90 ${ }_{\text {C }}$ (greenness chromatic coordinate) (Vrieling et al., 2018), etc. The uncertainties possibly existing in the processes of digital camera-based detections of tree defoliation phenology have also been exploited (Nagai et al., 2015). All of these endeavors indicated that camera-based plant phenology monitoring techniques can record the interannual variations of plant dynamics via continuously imaging, with both adequate temporal frequencies and high spatial resolutions. As such, camera-based phenology monitoring can bridge the gaps between satellite-collected phenological variables and field observation data. However, it is difficult to justify the installation and maintenance of cameras at multiple locations for phenology monitoring purposes in urban areas, and high municipal cost is the primary factor restricting the above-reviewed camera-based plans from being extended to accomplish the task of urban plant phenology monitoring.

Aiming at this technical gap, the present study attempted to expand the functions of the widespread surveillance cameras (SCs) in cities all over the world to urban plant phenology monitoring. In fact, using surveillance or security cameras for plant phenology observations has been reported in a few previous studies. For example, Petach et al. (2014) have validated an infrared-enabled security camera for plant phenology monitoring. However, the focus of the research was still on how to play the roles of such single cameras, rather than on how to improve the total performance of the SC networks, and thereby, such an application does not have fundamental differences with the endeavors based on the principle of repeating digital photography (Richardson et al., 2007; Crimmins and Crimmins, 2008; Ahrends et al., 2009; Graham et al., 2009; Ide and Oguma, 2010; Sonnentag et al., 2011; Hufkens et al., 2012; Zhao et al., 2012; Nijland et al., 2014; Nagai et al., 2015; Toomey et al., 2015; Alberton et al., 2017; Vrieling et al., 2018). Furthermore, plants in urban environments at local scales mostly are of high diversities. Hence, the general-purposed approaches proposed in the previous researches, e.g., tracking spring green-up in a deciduous broadleaf forest (Richardson et al., 2007), cannot effectively handle the targets lying in the fields of view of the SCs. In other words, plant-level rather than stand-level solutions for urban plant phenology monitoring are required. To handle these problems, the present study proposed a more systematic solution plan.

\section{Methods}

\subsection{Study site and data collection}

The chosen study site is located in the campus of Peking University, Beijing, China $\left(39^{\circ} 54^{\prime} 20^{\prime \prime} \mathrm{N}, 116^{\circ} 25^{\prime} 29^{\prime \prime} \mathrm{E}\right)$, characterized by a typical kind of warm and semi-humid continental monsoon climate. The campus covers 339 ha, with an average altitude of $56.9 \mathrm{~m}$. The phenological data for this case study were collected using a surveillance camera (DS-2AF1-784DS, Hikvision, China), which was installed (facing northwest) at a height of $4 \mathrm{~m}$ above the ground. The optical sensor 
of the camera was a $1 / 3^{\prime \prime}$ progressive scan complementary metal oxide semiconductor (CMOS), enclosed in a commercial waterproof housing. The mode of camera operation was set in an "automatic mode" for both exposure and white balance adjustment, similar to the mode used by Zhao et al. (2012). Although the camera was fixed, inevitable moves caused by external environmental influences such as winds and rains might cause changes in its field of views. To handle this issue, serial image registration (Lou et al., 2008) was conducted. Specifically, the SC image series were initially registered based on the approaches of Feature-Based Matching (FBM) and Area-Based Matching (ABM) and, then, fine registered using the epipolar geometric theory based algorithm that is based on Support Vector Machine (SVD) decomposition and weighted normalized Fundamental matrix (F-matrix) calculation (Lou et al., 2008). Next, to minimize the impacts possibly caused by different solar angles (e.g., Calders et al., 2015), the images recorded at about 7: 00 a.m. every day were selected for the phenological studies. The normalization of the Digital Number (DN) values was implemented based on the easily detectable and relatively consistent objects like impervious road surfaces. The observation lasted from March 27 to May 14,2015 , and the images were automatically saved in the format of Joint Photographic Experts Group (JPEG, $1920 \times 1080$ pixels resolution with three channels of 24-bit red-green-blue (RGB) color information).

The foreground of the camera was predominantly covered by ornamental plants, which are an important kind of plants of wide interest in phenological studies (e.g., Zhang et al., 2014). The plants selected for this case study include a black locust (Robinia pseudoacacia), a cherry plum (Prunus cerasifera) and a midget crabapple (Malus micromalus), with the distances to the camera being about $22 \mathrm{~m}, 25 \mathrm{~m}$ and $40 \mathrm{~m}$, respectively. The flowers of $R$. pseudoacacia and M. micromalus appear after their foliations begin (leaf-first), whereas the pink flowers of $P$. cerasifera tend to fall earlier than its purple leaves growing (flowerfirst). In addition, their greening processes are often disturbed by the prosperities of their background plants such as meadows. The scenarios mentioned above are the representative cases of spring ornamental plant phenologies, and this complexity also poses the challenges (Richardson et al., 2007) of detecting and determining the phenologies from the series of SC image records.

\subsection{Schematic plan}

The proposed schematic plan starts from analyzing the mechanism of SC operation. SCs are generally operated in such a way that multiple SCs are wire- or wireless-controlled by a Central Control Unit (CCU) (sometimes even just being a Personal Computer (PC)), and their data are wire- or wireless-transferred to a related CCU. Some of these controls are implemented through network connectivity such as internet. In light of this hardware-organization framework, the proposed schematic plan is to simply install an automatic software module with the function of plant phenology monitoring as a plug-in in those already-established RGB SC-related CCUs.

The software module comprises a series of functional units, involving image preprocessing on camera white balance identification and image registration, object segmentation, phenological index calculation, phenological modeling and phenophase output. The kernel of the software module is a general-purpose algorithm for determining the starting and ending dates of the key phenological events of differentspecies plants that stand in the field of view (FOV) of each wire-controlled SC. The bases of the algorithm mainly comprise derivation of plant phenological indices from the SC RGB channel DN values and then curve-fitting of the phenological phases for modeling of plant dynamics.

Unlike the single plant phenological event processing implemented in most studies using camera-based plant phenology tracking (e.g., Richardson et al., 2007; Crimmins and Crimmins, 2008; Ide and Oguma, 2010; Nijland et al., 2014; Vrieling et al., 2018), characterizing the phenological processes of diverse plants considered in this study becomes more complex. In such a case, the traditional algorithmic schemes such as sigmoid-shaped logistic function fitting (Richardson et al., 2007) become insufficient to capture the entire phenological phenomena. For this issue, the proposed schematic solution plan is to simultaneously detect and identify different phenological processes for different plant species lying in the same SC foreground within the same phase. This strategy is particularly necessary when post-processing the image records from a large number of SCs. That suggests that the successful findings of our work can be applied extensively wherever there are SCs. It should be acknowledged that phenological characterization is inherently complicated when multiple species and driving patterns are involved (Vrieling et al., 2018); thus, we tested the feasibility of the proposed solution using only representative scenarios.

\subsection{Algorithm development}

\subsubsection{Phenological indices}

Due to environmental disturbances such as sunlight variation, fog and rain, the original RGB features of the collected digital image series proved to be inappropriate to accurately reflect the real situations of canopy changes (Richardson et al., 2007). In addition, the popular temporal analyses of the DN series of each color channel might derive wrong phenological conclusions (Alberton et al., 2017). Two phenological indices that are able to avoid the above-mentioned influences were proposed. The first phenological index is Excessive Greenness Index (GEI), which has been widely used in previous works (e.g., Richardson et al., 2007; Nagai et al., 2015). GEI can enhance the signal from green plant material via making a distinction between green plants and background (Richardson et al., 2007; Nagai et al., 2015; Ahrends et al., 2008). Here, we used the sensitivity of GEI to green-band signal to track the emergence and decline of flowers. The second phenological index is Relative Channel Index (RCI), which is defined as the ratio between the DN recorded by each channel and the sum of the DNs for the R, G and B channels (denoted as $\mathrm{RCI}_{R}, \mathrm{RCI}_{\mathrm{G}}$ and $\mathrm{RCI}_{\mathrm{B}}$, respectively). The indices similar to the RCIs have been applied by Richardson et al. (2007) and Ahrends et al. (2008) to detect spring plant phenology.

The two phenological indices are defined as

$G E I=G-R+(G-B)=2 * G-(R+B)$

$R C I_{X}=X /(R+G+B)$

where R, G and B present the DN values for the R, G and B channels, and $X$ relates to the DN values for any R, G or B channel. The "relative" scheme in the forms of either "subtraction" or "division" can handle the ill scenario of interdiurnal inconsistency in channel DNs due to sunlight variations evidenced as follows. The interdiurnal differences of sunlight strengths are simultaneously sensed by different channels, and the "subtraction" or "division" operations are equal to removing such interdiurnal changes in a synchronous way. Then, the interdiurnal changes reflected by the "relative" results are exactly caused by plant dynamics, with different DN reflections by different SC color channels (Richardson et al., 2007).

\subsubsection{Phenological model}

The proposed phenological model needs to be able to implement the task of simultaneously detecting and identifying different phenological processes for different plant species lying in the same SC foreground within the same phase. As such, the proposed phenological model needs to be able to simultaneously derive multifold formulas of the proposed phenological indices to best characterize the different phenological processes within the observation phase, e.g. foliation, blossom, blossom and then withering away, defoliation and meadow greening (background change), possibly occurring within the SC foreground. For such complex scenarios, the traditional solution based on the bi-logistic models (Richardson et al., 2007; Ahrends et al., 2008) or many other 
kinds of species-specific phenological models (Chuine et al., 1998; Henneken et al., 2013; Zhang and Zhang, 2015; Xie et al., 2018) cannot work well. That is, the bi-logistic models appropriate for monitoring of leaf dynamics cannot be used for simultaneously monitoring of the flowering processes, because detection of the flowering phases is more complicated than only observing leaf color changes, e.g. the scenario that the pixels relating to plant flowers are easily blocked by the neighboring leaves and background. To address these issues, we proposed a novel multi-Gaussian model that is defined as

$\mathrm{g}(x)=\sum_{i=1}^{n} \mathrm{a}_{i} \mathrm{e}^{-\left(\frac{x-\mathrm{b}_{i}}{\mathrm{c}_{i}}\right)^{2}}$

where $x$ represents the day of year (DOY, January 1 is deemed as DOY $1), g(x)$ is the model that can best characterize the different phenological processes within a limited phase (e.g., two months here), and a, b and $c$ are the fitted coefficients. The values of $a, b$ and $c$ for different prominent levels ( $i$ ) of phenological phenomena are determined by pursuing the minimum result in fitting unconstrained multi-variable functions against the temporal series of the calculated phenological indices. This is implemented based on the least-square fitting method (Ahrends et al., 2008). Given that the model is derived within a limited phase, the method for plant phenological modeling proposed in this study can be denoted as the phase-limited multi-Gaussian phenological model.

Based on the fitted multi-Gaussian model, the key phenological dates such as the beginning or ending of blossoms can be determined using the traditional thresholding method (Ahrends et al., 2008). For example, for each Gaussian curve that corresponds to the blossom process, the DOY when the phenological index reaches a certain percentage of its maximum amplitude is defined as the start of the flowering period (SFP), and the end of the flowering period (EFP) is determined when the phenological index reduces to the same threshold value (Ahrends et al., 2008; Nagai et al., 2015). The same procedure can be used to determine the start of the foliating period and the end of the foliating period.

\subsection{Performance assessment}

In order to precisely assess the performance of the phenological indices and the phenological models proposed in this study, the SC serial data collected for the case study were processed. Since the field of view of the SC changed inadvertently, image registration was firstly executed using the ENVI (ENvironment for Visualizing Images) Software for the purpose of implementing the consecutive analysis of the digital images. Then, we manually drew three fixed regions of interest (ROIs) to represent the crowns of the P. cerasifera (ROI A), $M$. micromalus (ROI B) and R. pseudoacacia (ROI C). The drawing of the ROIs followed the rule of maintaining a compromise between optimizing the performance of crown representations and avoiding the interferences from environments as much as possible. At last, the data for performance assessment were obtained. The course of SC recording of the $P$. cerasifera and M. micromalus lasted from March 27 to April 28, 2015, while the course for the R. pseudoacacia was from April 23 to May 14, 2015.

Then, performance assessment was finished as follows. The SFPs and EFPs of the three prominent plants of different species lying in the foreground of the SC were derived and compared to their reference values. The ground-truth values were acquired via visual observation of SC image series, which has been widely used as a general knowledgebased verification tool for camera-based phenological identification (Ahl et al., 2006; Lin and West, 2016). Three independent observers used a common protocol to define the following dates for each plant: 1) When the three crowns started to present flowers; 2) When the majority of flowers withered away. The transitional dates of the phenological developments were determined to be their SFPs and EFPs. The SFPs and

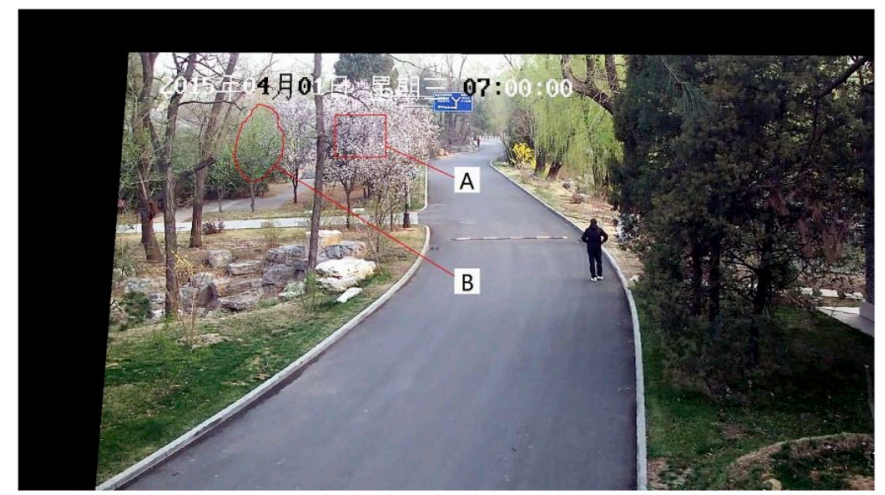

(a)

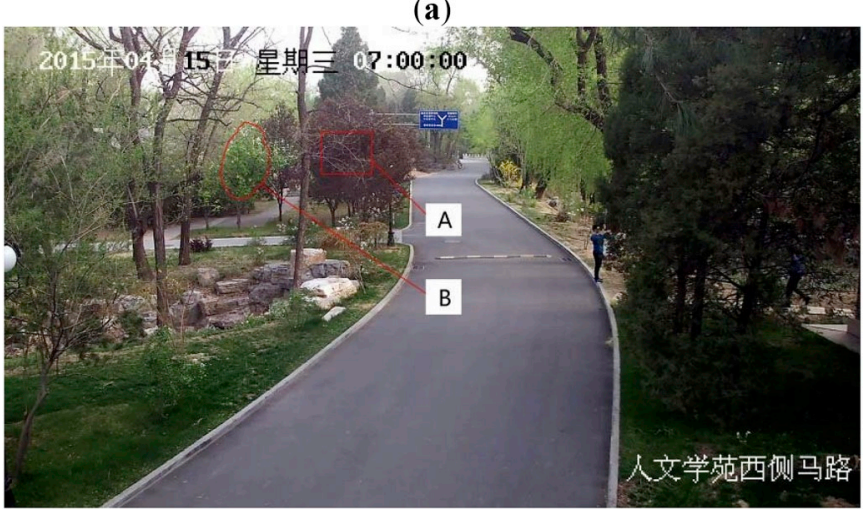

(b)

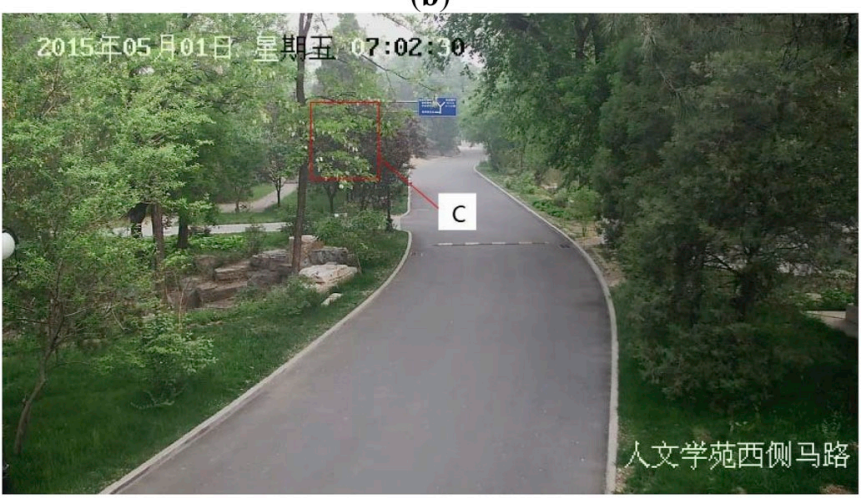

(c)

Fig. 1. Illustrations of the SC images after registration and the three ROIs drawn for segmenting the sample plants (the Chinese characters stamped in the SC images at their bottom-right corners mean the location of the SC and those stamped characters at the top-left corners mean the time of recording the images).

EFPs derived from the assumed phenological model and visual interpretation were compared to show the performance of the used phenological indices and the proposed phenological model.

\section{Results}

\subsection{The processed data}

The SC images collected at 7:00 a.m. were isolated for the test. Then, the series of the SC images were firstly registered, since the foreground of the SC might show minor orientation shifts due to target searching or wind blowing. Next, the ROIs for characterizing the crowns of $P$. cerasifera (ROI A), M. micromalus (ROI B) and R. pseudoacacia (ROI C) were drawn, as indicated in Fig. 1. The following analyses were carried out based on the resulting ROI series individually for the three sample plants. 


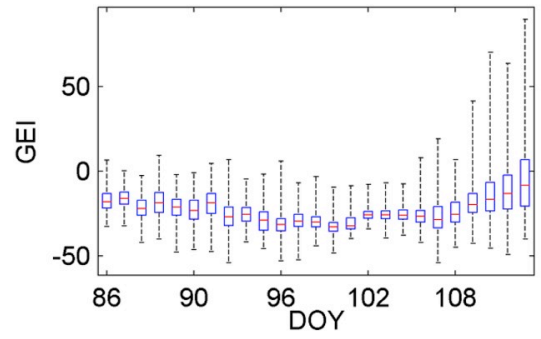

(a)

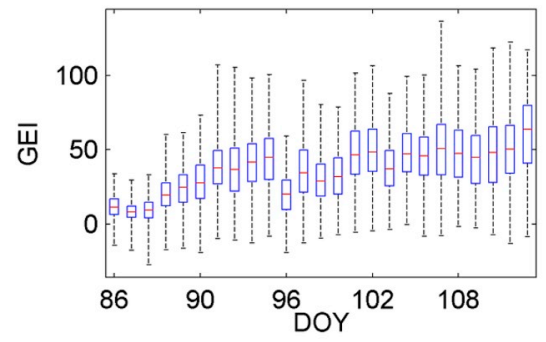

(c)

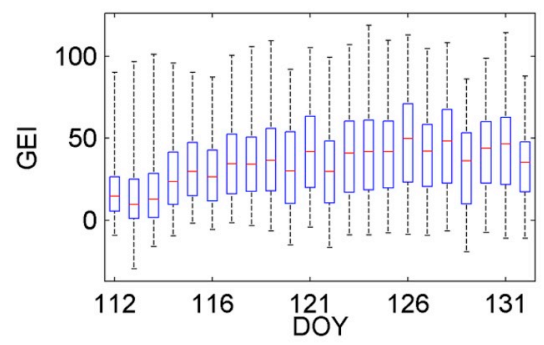

(e)

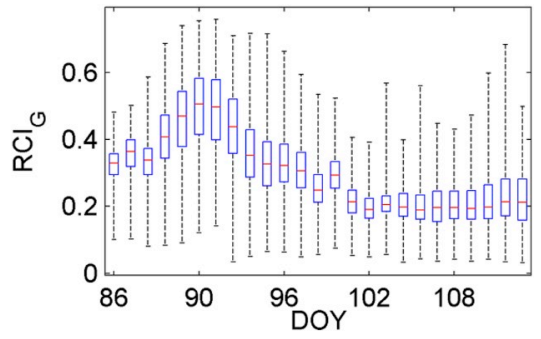

(b)

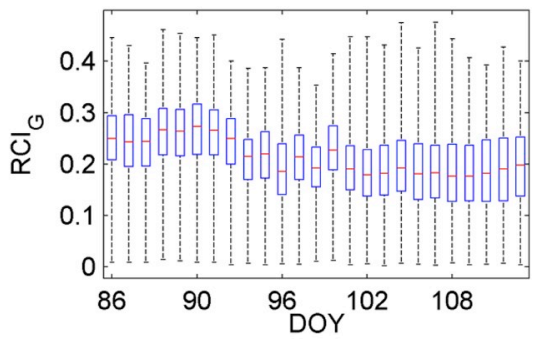

(d)

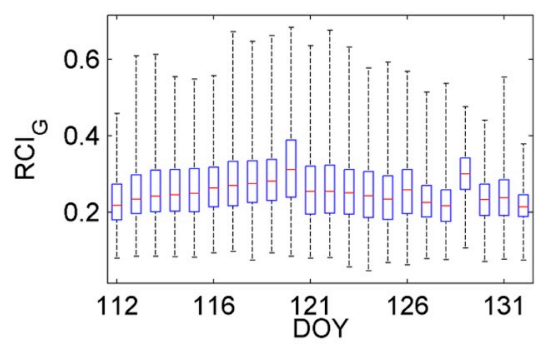

(f)

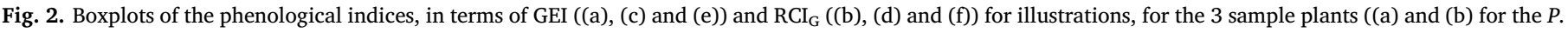
cerasifera, (c) and (d) for the M. micromalus, and (e) and (f) for the R. pseudoacacia).

A comparison with the field observations proved that the registered image series can be used by people to identify plant phenological processes. Although back- or repeated-checking was often needed, most observers of these images could tell the following stories. The ROI of the $P$. cerasifera (ROI A) in its full-blossom phase was dominated by its pink flowers. The ROI of the M. micromalus (ROI B) in its foliating phase was mixed with some pink flowers of another $P$. cerasifera (Fig. 1a). Then, the leaves of the $M$. micromalus became larger and greener so that the pink flowers behind it were obstructed. Next, the ROI of the $M$. micromalus appeared obviously with a number of its white flowers but was still filled with its green leaves. Synchronously, the pink flowers of the $P$. cerasifera withered gradually, and the ROI of the P. cerasifera was increasingly occupied by its purple leaves and brown branches (Fig. 1b). Subsequently, the ROI of the P. cerasifera (ROI A) was gradually obstructed by the flourishing crown of the R. pseudoacacia that is closer to the SC. This triggered the effect of overlapping between the ROI C ( $R$. pseudoacacia) and the ROI A ( $P$. cerasifera). As regards $R$. pseudoacacia (Fig. 1C), the beginning of its foliation was earlier than the start of its flowering, and this phenomenon rendered leaves to play a major role in the ROI C. It was also realized that the blossom process of the $P$. cerasifera occurred within a short time, the phase for ROI C phenological analysis needed to be shortened in order to minimize the disturbances possibly caused by the $P$. cerasifera (in the $R$. pseudoacacia's background in the FOV of the SC) with a different phenological behaving mode. This could explain the necessity of introducing the strategy of characterizing different phenological processes within a limited phase.

\subsection{The derived phenological indices}

Based on the extracted ROI series, the proposed phenological indices were derived (illustrated in Fig. 2). It can be recognized that for the same plants, the two phenological indices (GEI and RCI) performed with different patterns, regarding their features of no matter mean, maximum, minimum or value range. It can also be noticed that some of the resulting patterns, somehow, can possibly reflect the phenological processes. For example, it seems that the serial boxplots of $\mathrm{RCI}_{\mathrm{G}}$ for the $P$. cerasifera can characterize its blossom phase (Fig. 2b). Specifically, the $\mathrm{RCI}_{\mathrm{G}}$ firstly presented an apparent trend of value increasing along with the development of flowering; then, after reaching the peak, the $\mathrm{RCI}_{\mathrm{G}}$ declined day by day and eventually went to a stable status after about the DOY 102. The latter part relates to the phase that its pink flowers went withering and its purple leaves were growing.

\subsection{The derived phenological models}

The phenological models for the $P$. cerasifera, $M$. micromalus and $R$. pseudoacacia were derived in the phases of DOY 88 to DOY 99, DOY 99 to DOY 111 and DOY114 to DOY125, respectively (Fig. 3). The three primary Gaussian curves of the resulting multi-Gaussian models are shown in Fig. 3a, c and 3e, and the Gaussian curves relating to the blossom phases for the P. cerasifera, M. micromalus and R. pseudoacacia are shown in Fig. 3b, d and 3f, respectively. Further, based on the identified Gaussian curves relating to the blossom processes, the SFPs and EFPs were determined in terms of the optimal thresholds. Table 1 presents the scheme of pursuing the optimal threshold values. That is, the SFPs and EFPs derived by setting uniform thresholds for different 


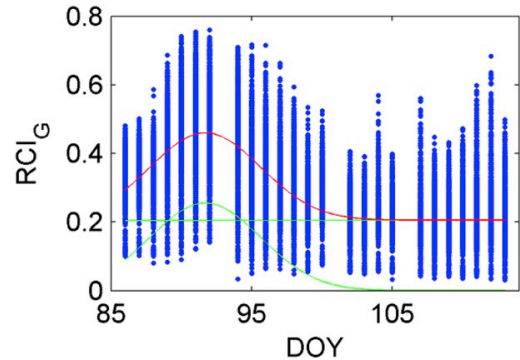

(a)

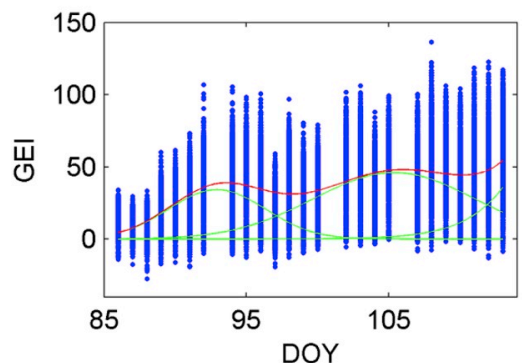

(c)

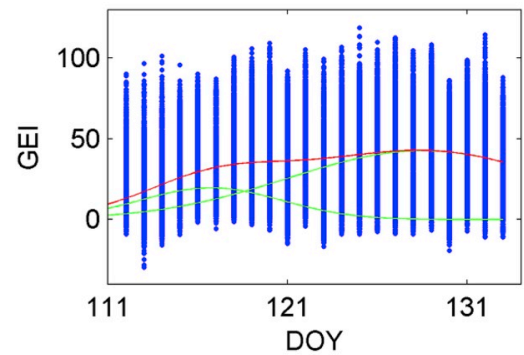

(e)

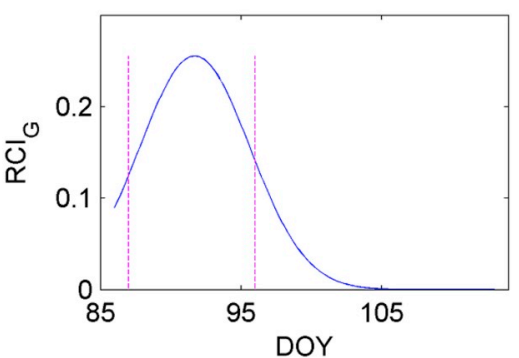

(b)

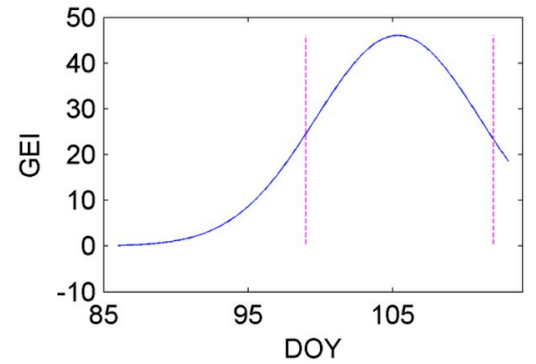

(d)

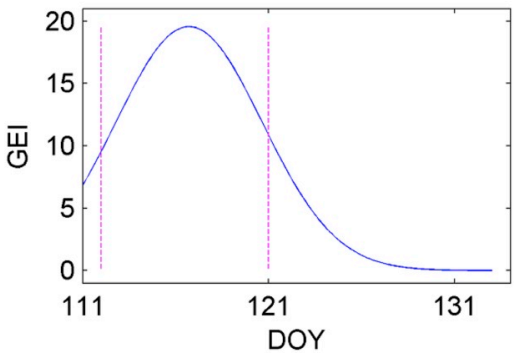

(f)

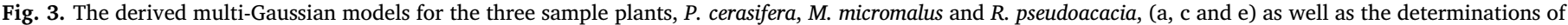

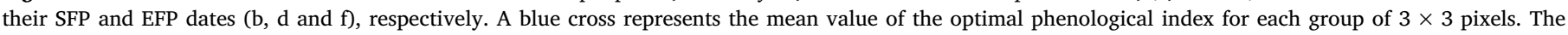

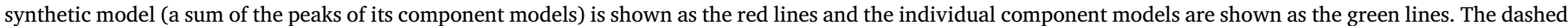
lines in (b, $d$ and $f$ ) indicate the SFP and EFP dates.

Table 1

Threshold determination by seeking the best agreements of both SFP and EFP for all of the plants for the test. Obs: the dates determined by human observation of the SC image series; SFP: the start of the flowering period; EFP: the end of the flowering period. All dates are in the form of DOY (day of year).

\begin{tabular}{|c|c|c|c|c|c|c|c|c|}
\hline & \multicolumn{2}{|c|}{ P. cerasifera } & \multicolumn{2}{|c|}{ M. micromalus } & \multicolumn{2}{|c|}{ R. pseudoacacia } & \multirow[t]{2}{*}{$\mathrm{R}^{2}$} & \multirow[t]{2}{*}{ RMSE } \\
\hline & SFP & EFP & SFP & $E F P$ & SFP & $E F P$ & & \\
\hline Obs & 88 & 99 & 99 & 111 & 114 & 125 & & \\
\hline $10 \%$ & 83 & 100 & 93 & 113 & 108 & 125 & 0.9436 & 4.007 \\
\hline $20 \%$ & 84 & 99 & 95 & 113 & 110 & 124 & 0.9687 & 3.035 \\
\hline $30 \%$ & 85 & 98 & 97 & 113 & 111 & 123 & 0.9803 & 2.200 \\
\hline $40 \%$ & 86 & 97 & 98 & 113 & 111 & 122 & 0.9811 & 1.927 \\
\hline $50 \%$ & 87 & 96 & 99 & 112 & 112 & 122 & 0.9861 & 1.380 \\
\hline $60 \%$ & 87 & 96 & 100 & 111 & 113 & 121 & 0.9848 & 1.538 \\
\hline $70 \%$ & 88 & 95 & 101 & 111 & 113 & 120 & 0.9731 & 1.737 \\
\hline $80 \%$ & 89 & 94 & 102 & 109 & 114 & 120 & 0.9593 & 2.134 \\
\hline $90 \%$ & 90 & 93 & 103 & 108 & 115 & 119 & 0.9289 & 3.129 \\
\hline
\end{tabular}

plants were compared to those acquired by human observations of the SC image series, and the minimal divergences related to the optimal thresholds. For the three sample plants, the threshold relating to $50 \%$ for all of the maxima of the Gaussian curves was determined (see Table 1 and Fig. 3). When this scheme is assumed by other users, it is noted that the determination of the thresholds needs to be conducted based on a portion of the plants within the FOV of the SC for training and the remaining for test.
Table 2

Derivation of the key phenological dates for the 3 sample plants and the performance acquired by comparing the estimated dates (Est) with the manuallyobserved dates from the images (Obs), i.e., in terms of their difference (Diff). All dates are in the form of DOY (day of year). SFP: the start of the flowering period; EFP: the end of the flowering period. FPL: the flowering period length.

\begin{tabular}{llllllllll}
\hline & \multicolumn{1}{c}{ P. cerasifera } & \multicolumn{4}{c}{ M. micromalus } & \multicolumn{3}{c}{ R. pseudoacacia } \\
\cline { 2 - 9 } & SFP & EFP & FPL & SFP & EFP & FPL & SFP & EFP & FPL \\
\hline Obs & 88 & 99 & 11 & 99 & 111 & 12 & 114 & 125 & 11 \\
Est & 87 & 96 & 9 & 99 & 112 & 13 & 112 & 122 & 10 \\
Diff & -1 & -3 & -2 & 0 & 1 & 1 & -2 & -3 & -1
\end{tabular}

\subsection{Performance}

The performance of the proposed phenological indices and phenological models is shown in Table 2. Compared to the manually-observed phenological dates, the estimations showed quite less divergence. For the three sample plants, the maximum error is three days. This also indicated that the proposed schematic plan for phenological analysis is available not only for different plants but also for their different phenological processes. The results have basically validated the proposed solution. 


\section{Discussions}

\subsection{Influence factors}

The first factor possibly impacting the performance of a SC in urban plant phenology monitoring is originating from environmental disturbance that can deteriorate the derivation of the prescribed phenological indices. For instance, fog and ill atmospheric conditions may create noise in phenological indices (Richardson et al., 2007; Bradley and Clarke, 2011; Nagai et al., 2015). Ide and Oguma (2010) found that it is necessary to apply data filtering to reduce the influence of varying weather conditions. In this study, the SC was set to be able to automatically keep white balance, but the influence caused by different fog and sun illumination still existed in the processed SC image series and, subsequently, in the resulting key phenological dates. The mature techniques of image enhancement as discussed in (Bradley and Clarke, 2011) can be merged into the plug-in in a follow-up study. It is also worth exploring other plans, e.g., dark pixel subtraction, illumination normalization according to the extraterrestrial irradiance of the color bands divided by the cosine of the sun zenith, and statistics-based view angle correction by taking the solar position into account, for dealing with the possible influence factors.

The second influence factor is the instability of its FOV. In addition to wind and rain, human operation is another inevitable cause of SC FOV changes. After all, SC cameras are installed with the main purpose of security surveillance. For better observing the locations of interest, SC operators often need to shift the FOV, sometimes even out of the often-observed ones, and occasionally even forget to adapt them back. These actions may lead to a series of uncontinuous image recordings and even two totally-different sub-series of image recordings. Such data certainly result in higher errors in the estimation of key phenological dates. The phase-limited phenological modeling solution plan proposed in the present study can handle this problem to some extent but still cannot overcome it completely.

The analyses conducted in this study manifested that crown overlapping in the SC foreground is another factor possibly deteriorating the phenological derivations. In some cases, this factor may lead to more serious errors in phenological information extraction than due to FOV changes. This outcome is more certain in the case of a single series of image recordings with an ensemble of two or more plant species lying in the SC's FOV create complex canopy structures (Calders et al., 2015), compared to assessing two separated image recordings of different targets. The plants behind the targeted plants even possibly play a primary role in the FOV, and the initial physiological stages of the SCfocused plants may be overlapped. This scenario may be a potential shortcoming of the proposed multi-Gaussian phenological model.

Species heterogeneity is also an important influence factor in expanding the functions of SCs to nature rhythm understanding. In addition to the aforementioned effect of crown overlapping, weak capability of SCs in species representation can be caused by species heterogeneity for different regions of interest. Generally, the small FOV of urban SCs allows imaging only a few plants, and thus certain species might be under-sampled, resulting in unreliable/inconclusive phenological information derivation. That is, an individual plant surrounded by the plants of different species often suffers some physiological or competitive disturbances, and consequently, their phenological performance may be out of their normal ranges. It is worth mentioning that the derived phenological dates may be misleading, and thereby, it might be necessary to build confidence interval as a range of potential dates.

\subsection{Further plans}

For the purpose of developing a kind of fully-functional automatic software plug-in for urban SC-based plant phenology monitoring, more work need to be done in the next studies. The first plan is to introduce the methods developed for plant detection and segmentation in various point-of-view images, particularly for those collected in the oblique or horizontally imaging modes. In such special scenarios, the algorithm of unmanned aerial vehicle oblique image-based individual tree recognition proposed in (Lin et al., 2015) can be applied for the purpose of detecting individual plants in the FOV of the relatively highly-mounted SCs. The procedures of automatic estimation of tree diameters (Yi and Moon, 2015) can be applied to plant isolation in the images of approximately-horizontal SC FOV. High-performance plant segmentation in SC images can logically improve the characterization of plant morphology and, hence, a more accurate derivation of plant phenological information.

The second measure is to derive and combine multiple plant phenological indices, each performing its best at its nominal scale. The combination of the spectral shape indices posed for satellite imagebased plant phenological analyses (Palacios-Orueta et al., 2012), the GEI calculated for Webcam-based floral phenological observations (Richardson et al., 2007), and the Visible Atmospherically Resistant Index (VARI) that can compensate for additional blue light due to dawn or overcast sky (Gitelson et al., 2002) can be attempted in following study. The most powerful phenological indices or the weights for counting each of such prescribed phenological indices in phenological modeling can be detected, e.g., by using the method of comparing the divergence of different phenological index values. By this way, the issue that merely observing a limited number of phenological indices being insufficient for examining key phenological processes can be avoided to some extent.

The third plan is to assume multiple phenological models in an integrative way to overcome the common problem of incorrect estimation of key phenological dates. In fact, different phenological models may work well for different kinds of phenological processes in the different urban and natural environments. The most common case is the doublesigmoid phenological model as used in (Zavalloni et al., 2006) and the UniChill phenological model assumed in (Chuine, 2000). Combining such different phenological models and even newly developing more powerful models of consistently reflecting urban and natural phenological characteristics can be useful for both a holistic characterization and a multi-level analysis of phenological process, and the phenologyrelated results can be obtained in a more comprehensive way, ultimately turning into global phenology monitoring networks.

In addition to the above-mentioned technical plans, some other improvements can also be introduced to add the efficiency of the installed software plug-ins. For instance, although the proposed algorithm was designed for the general-purpose usages from the beginning, the targeted plants within the FOV of each SC need to be pre-determined. This can be operated during the installing and adapting of the software plug-in in each CCU. This will permit the technicians and operators of these plug-ins to simultaneously examine the FOVs of the SCs, identify the species of the SC-focused plants, and adapt the set-ups of the SCs by following their kernel properties; after these pre-treatments, more appropriate functional modes can be set to better monitor the phenological processes. The functionality of such software modules can also be periodically checked via internet and modified as necessary. With such measures integrated, the developed module may serve as a reliable and efficient medium of expanding the functions of urban surveillance cameras to understanding of ecosystems services such as, Arctic vegetation phenology (Anderson et al., 2016), urbanization effect on plant flowering phenology (Neil and Wu, 2006), and urban heat island influence on land surface phenology (Krehbiel et al., 2016).

\section{Conclusion}

Although urban SCs often face more complex scenes typically with more plant species than the already validated phenology-purposed webcams, they proved to work for urban plant phenology monitoring in the present study. In the case of three plants with their foliation and 
flowering mixed together, the tests showed that the proposed phaserestricted multi-Gaussian phenological models can handle the issue of signal mixture for different phenological processes of different plant species. That is, the applicability of the widespread RGB-typed SCs for urban plant phenology monitoring has been basically validated. The significance of this study and its findings is that, the solution can be extended to composing plant phenology observation networks at different scales, from city to region, continent and even the globe, for better understanding of the natural rhythms of terrestrial ecosystems and the inherent mechanisms of vegetation-climate interactions.

\section{Ethical statement}

Both the authors and the paper follow the related ethical principles.

\section{Conflicts of interest}

Both the authors and the paper have no conflict of interest.

\section{Acknowledgements}

This work was financially supported in part by the National Natural Science Foundation of China (Grant No. 31670718 and 31870531) and in part by the SRF for ROCS, SEM, China.

\section{Appendix A. Supplementary data}

Supplementary data to this article can be found online at https:// doi.org/10.1016/j.rsase.2019.05.001.

\section{References}

Abu-Asab, M.S., Peterson, P.M., Shetler, S.G., Orli, S.S., 2001. Earlier plant flowering in spring as a response to global warming in the Washington, DC, area. Biodivers. Conserv. 10, 597-612.

Ahl, D.E., Gower, S.T., Burrows, S.N., Shabanov, N.V., Myneni, R.B., Knyazikhin, Y., 2006. Monitoring spring canopy phenology of a deciduous broadleaf forest using MODIS. Remote Sens. Environ. 104, 88-95.

Ahrends, H.E., Brügger, R., Stöckli, R., Schenk, J., Michna, P., Jeanneret, F., Wanner, H., Eugster, W., 2008. Quantitative phenological observations of a mixed beech forest in northern Switzerland with digital photography. J. Geophys. Res.: Biogeosciences 113.

Ahrends, H.E., Etzold, S., Kutsch, W.L., Stoeckli, R., Bruegger, R., Jeanneret, F., Wanner, H., Buchmann, N., Eugster, W., 2009. Tree phenology and carbon dioxide fluxes: Use of digital photography for process-based interpretation at the ecosystem scale. Clim. Res. 39, 261-274.

Alberton, B., Torres, R.S., Cancian, L.F., Borges, B.D., Almeida, J., Mariano, G.C., dos Santos, J., Morellato, L.P.C., 2017. Introducing digital cameras to monitor plant phenology in the tropics: Applications for conservation. Perspect. Ecol. Conserv. 15, 82-90.

Anderson, H.B., Nilsen, L., Tømmervik, H., Karlsen, S.R., Nagai, S., Cooper, E.J., 2016. Using ordinary digital cameras in place of near-infrared sensors to derive vegetation indices for phenology studies of high Arctic vegetation. Rem. Sens. 8, 847.

Aono, Y., Saito, S., 2010. Clarifying springtime temperature reconstructions of the medieval period by gap-filling the cherry blossom phenological data series at Kyoto, Japan. Int. J. Biometeorol. 54, 211-219.

Baldocchi, D., Black, T., Curtis, P., Falge, E., Fuentes, J., Granier, A., et al., 2005. Predicting the onset of net carbon uptake by deciduous forests with soil temperature and climate data: A synthesis of FLUXNET data. Int. J. Biometeorol. 49, 377-387.

Bradley, E.S., Clarke, K.C., 2011. Outdoor webcams as geospatial sensor networks: Challenges, issues and opportunities. Cartogr. Geogr. Inf. Sci. 38, 3-19.

Calders, K., Schenkels, T., Bartholomeus, H., Armston, J., Verbesselt, J., Herold, M., 2015. Monitoring spring phenology with high temporal resolution terrestrial LiDAR measurements. Agric. For. Meteorol. 203, 158-168.

Chuine, I., 2000. A unified model for budburst of trees. J. Theor. Biol. 207, 337-347.

Chuine, I., Cour, P., Rousseau, D., 1998. Fitting models predicting dates of flowering of temperate-zone trees using simulated annealing. Plant Cell Environ. 21, 455-466.

Churkina, G., Schimel, D., Braswell, B.H., Xiao, X., 2005. Spatial analysis of growing season length control over net ecosystem exchange. Glob. Chang. Biol. 11, $1777-1787$.

Comber, A., Brunsdon, C., 2015. A spatial analysis of plant phenophase changes and the impact of increases in urban land use. Int. J. Climatol. 35, 972-980.

Crimmins, M., Crimmins, T.M., 2008. Monitoring plant phenology using digital repeat photography. Environ. Manag. 41, 949-958.

Dai, J., Wang, H., Ge, Q., 2013. Multiple phenological responses to climate change among 42 plant species in Xi'an, China. Int. J. Biometeorol. 57, 749-758.

Eklundh, L., Jönsson, P., 2015. TIMESAT: A software package for time-series processing and assessment of vegetation dynamics. Rem. Sens. Digit. Image Process. 22, $141-158$.

Ganguly, S., Friedl, M.A., Tan, B., Zhang, X., Verma, M., 2010. Land surface phenology from MODIS: Characterization of the collection 5 global land cover dynamics product. Remote Sens. Environ. 114, 1805-1816.

Gitelson, A.A., Kaufman, Y.J., Stark, R., Rundquist, D., 2002. Novel algorithms for remote estimation of vegetation fraction. Remote Sens. Environ. 80, 76-87.

Gonsamo, A., Chen, J., D'Odorico, P., 2013. Deriving land surface phenology indicators from $\mathrm{CO}_{2}$ eddy covariance measurements. Ecol. Indicat. 29, 203-207.

Gonsamo, A., Chen, J., Wu, C., Dragoni, D., 2012. Predicting deciduous forest carbon uptake phenology by upscaling FLUXNET measurements using remote sensing data. Agric. For. Meteorol. 165, 127-135.

Graham, E.A., Yuen, E.M., Robertson, G.F., Kaiser, W.J., Hamilton, M.P., Rundel, P.W., 2009. Budburst and leaf area expansion measured with a novel mobile camera system and simple color thresholding. Environ. Exp. Bot. 65, 238-244.

Henneken, R., Dose, V., Schleip, C., Menzel, A., 2013. Detecting plant seasonality from webcams using bayesian multiple change point analysis. Agric. For. Meteorol. 168, 177-185.

Hufkens, K., Friedl, M., Sonnentag, O., Braswell, B.H., Milliman, T., Richardson, A.D., 2012. Linking near-surface and satellite remote sensing measurements of deciduous broadleaf forest phenology. Remote Sens. Environ. 117, 307-321.

Ide, R., Oguma, H., 2010. Use of digital cameras for phenological observations. Ecol. Inf. $5,339-347$.

Jochner, S., Alves-Eigenheer, M., Menzel, A., Morellato, L.P.C., 2013. Using phenology to assess urban heat islands in tropical and temperate regions. Int. J. Climatol. 33, 3141-3151.

Jochner, S., Menzel, A., 2015. Urban phenological studies - past, present, future. Environ. Pollut. 203, 250-261.

Jochner, S., Sparks, T., Estrella, N., Menzel, A., 2012. The influence of altitude and urbanization on trends and mean dates in phenology (1980-2009). Int. J. Biometeorol 56, 387-394.

Koerner, C., Basler, D., 2010. Phenology under global warming. Science 327, 1461-1462.

Krehbiel, C.P., Jackson, T., Henebry, G., 2016. Web-enabled Landsat data time series for monitoring urban heat island impacts on land surface phenology. IEEE J. Sel. Top. Appl. Earth Obs. Remote Sens. 9, 2043-2050.

Lafleur, P., Moore, T.R., Poon, D., Seaquist, J., 2005. Ecosystem phenology from eddycovariance measurement: Spring photosynthesis in a cool temperate bog. AGU Fall Meet 2005.

Lin, Y., Jiang, M., Yao, Y., Zhang, L., Lin, J., 2015. Use of UAV oblique imaging for the detection of individual trees in residential environments. Urban For. Urban Green. 14, 404-412.

Lin, Y., West, G., 2016. Reflecting conifer phenology using mobile terrestrial LiDAR: A case study of Pinus sylvestris growing under the Mediterranean climate in Perth, Australia. Ecol. Indicat. 70, 1-9.

Lou, L., Zhang, F.-M., Xu, C., Li, F., Xue, M.-G., 2008. Automatic registration of aerial image series using geometric invariance. Proc. IEEE Inter. Conf. Autom. Logist. 1198-1203 Qingdao, China.

Lu, P., Yu, Q., Liu, J., Lee, X., 2006. Advance of tree-flowering dates in response to urban climate change. Agric. For. Meteorol. 138, 120-131.

Nagai, S., Inoue, T., Ohtsuka, T., Yoshitake, S., Nasahara, K., Saitoh, T., 2015. Uncertainties involved in leaf fall phenology detected by digital camera. Ecol. Inf. 30, 124-132.

Neil, K., Landrum, L., Wu, J., 2010. Effects of urbanization on flowering phenology in the metropolitan phoenix region of USA: Findings from herbarium records. J. Arid Environ. 74, 440-444.

Neil, K., Wu, J., 2006. Effects of urbanization on plant flowering phenology: A review. Urban Ecosyst. 9, 243-257.

Nijland, W., de Jong, R., de Jong, S., Wulder, M., Bater, C., Coops, N., 2014. Monitoring plant condition and phenology using infrared sensitive consumer grade digital cameras. Agric. For. Meteorol. 184, 98-106.

Palacios-Orueta, A., Huesca, M., Whiting, M., Litago, J., Khanna, S., Garcia, M., Ustin, S., 2012. Derivation of phenological metrics by function fitting to time-series of spectral shape indexes AS1 and AS2: Mapping cotton phenological stages using MODIS time series. Remote Sens. Environ. 126, 148-159.

Petach, A., Toomey, M., Aubrecht, D., Richardson, A., 2014. Monitoring vegetation phenology using an infrared-enabled security camera. Agric. For. Meteorol. 195, $143-151$.

Richardson, A.D., Jenkins, J.P., Braswell, B.H., Hollinger, D.Y., Ollinger, S.V., Smith, M.L., 2007. Use of digital webcam images to track spring green-up in a deciduous broadleaf forest. Oecologia 152, 323-334.

Rutishauser, T., Luterbacher, J., Jeanneret, F., Pfister, C., Wanner, H., 2007. A phenology-based reconstruction of interannual changes in past spring seasons. J. Geophys. Res.: Biogeosciences 112.

Sonnentag, O., Detto, M., Vargas, R., Ryu, Y., Runkle, B., Kelly, M., Baldocchi, D., 2011. Tracking the structural and functional development of a perennial pepperweed (lepidium latifolium 1.) infestation using a multi-year archive of webcam imagery and eddy covariance measurements. Agric. For. Meteorol. 151, 916-926.

Studer, S., Stockli, R., Appenzeller, C., Vidale, P.L., 2007. A comparative study of satellite and ground-based phenology. Int. J. Biometeorol. 51, 405-414.

Toomey, M., Friedl, M.A., Frolking, S., Hufkens, K., Klosterman, S., Sonnentag, O. Baldocchi, D., et al., 2015. Greenness indices from digital cameras predict the timing and seasonal dynamics of canopy-scale photosynthesis. Ecol. Appl. 25, 99-115.

Verbesselt, J., Hyndman, R., Newnham, G., Culvenor, D., 2010. Detecting trend and seasonal changes in satellite image time series. Remote Sens. Environ. 114, 106-115.

Vrieling, A., Meroni, M., Darvishzadeh, R., Skidmore, A.K., Wang, T., Zurita-Milla, R., Oosterbeek, K., O'Connor, B., Paganini, M., 2018. Vegetation phenology from 
Sentinel-2 and field cameras for a Dutch barrier island. Remote Sens. Environ. 215, 517-529.

Wolkovich, E.M., Cook, B.I., Allen, J.M., Crimmins, T.M., Betancourt, J.L., Travers, S.E., Pau, S., Regetz, J., Davies, T.J., Kraft, N.J., et al., 2012. Warming experiments underpredict plant phenological responses to climate change. Nature 485, 494-497.

Wu, X., Liu, H., 2013. Consistent shifts in spring vegetation green-up date across temperate biomes in China, 1982-2006. Glob. Chang. Biol. 19, 870-880.

Xie, Y., Civco, D.L., Silander, J.A., 2018. Species-specific spring and autumn leaf phenology capture by time-lapse digital cameras. Ecosphere 9, e02089.

Yi, F., Moon, I., 2015. Automatic calculation of tree diameter from stereoscopic image pairs using digital image processing. Appl. Opt. 51, 4120-4128.

Zavalloni, C., Andresen, J., Flore, J., 2006. Phenological models of flower bud stages and fruit growth of 'Montmorency' sour cherry based on growing degree-day accumulation. J. Am. Soc. Hortic. Sci. 131, 601-607.
Zhang, A., 2014. Applicability analysis of phenological models in the flowering time prediction of ornamental plants in Beijing area. J. Appl. Meteorol. Sci. 25, 483-492.

Zhang, A., Zhang, J., 2015. Prediction of first flowering date of prunus discoidea in Beijing Yuyuantan Park using phenologica model. Meteorol. Sci. Technol. 43.

Zhang, X., Friedl, M.A., Schaaf, C.B., Strahler, A.H., 2004. Climate controls on vegetation phenological patterns in northern mid-and high latitudes inferred from MODIS data. Glob. Chang. Biol. 10, 1133-1145.

Zhang, X., Friedl, M.A., Schaaf, C.B., Strahler, A.H., Hodges, J.C., Gao, F., Reed, B.C., Huete, A., 2003. Monitoring vegetation phenology using MODIS. Remote Sens. Environ. 84, 471-475.

Zhao, J., Zhang, Y., Tan, Z., Song, Q., Liang, N., Yu, L., Zhao, J., 2012. Using digital cameras for comparative phenological monitoring in an evergreen broad-leaved forest and a seasonal rain forest. Ecol. Inf. 10, 65-72. 